\title{
Amnioinfusion and Bed Rest May Effectively Improve the Insufficient Circulation of the Umbilical Cord in Pregnant Women With Hyper-Coiled Cord and Oligohydramnios
}

\author{
Masamitsu Nakamura $^{\mathrm{a}, \mathrm{b}}$, Junichi Hasegawa ${ }^{\mathrm{a}}$, Hiroko Takita ${ }^{\mathrm{a}}$, Akihiko Sekizawa ${ }^{\mathrm{a}}$
}

\begin{abstract}
We experienced two cases with hyper-coiled cord in which fetal-umbilical-placental circulation was improved after amnioinfusion or bed rest. Therefore, amnioinfusion and bed rest to reduce the compression of the vulnerable umbilical cord in cases of hyper-coiled cord might improve the pathologic fetal-umbilical-placental circulation.
\end{abstract}

Keywords: Amnioinfusion; Oligohydramnios; Hyper-coiled cord; Umbilical circulation

\section{Introduction}

Hyper-coiled umbilical blood vessels are more vulnerable to kinking, compression, traction and torsion than normal coiled cord. Hyper-coiled cord tends to lead to fetal hypoxia due to an external force such as labor or a fetal movement more often than a normal coiled cord [1]. Several studies have shown that hyper-coiled cords are correlated with poor perinatal outcomes such as low birth weight, meconium staining of amniotic fluid at birth and fetal growth restriction [2-4]. In our previous study of intrauterine fetal death after the second trimester, fetal death was found to be caused by a hyper-coiled cord in $25.9 \%(21 / 81)$ of cases.

Since amniotic fluid is derived from fetal urine and the volume reflects the fetal-umbilical-placental circulation, oligohydramnios without the rupture of membranes (ROM) after the second trimester is recognized as the critical stage of fetal circulation [5]. The umbilical circulation may be adversely affected by external forces caused by uterine contractions or fetal movement in oligohydramnios. Therefore, in cases with

Manuscript submitted September 8, 2020, accepted September 16, 2020

Published online November 18, 2020

aDepartment of Obstetrics and Gynecology, Showa University School of Medicine, Tokyo, Japan

${ }^{b}$ Corresponding Author: Masamitsu Nakamura, Department of Obstetrics and Gynecology, Showa University School of Medicine, 1-5-8 Hatanodai, Shinagawa-ku, Tokyo 142-8666, Japan. Email: mashamitsu1975@gmail.com

doi: https://doi.org/10.14740/jmc3581 both a hyper-coiled cord and oligohydramnios, intervention should be considered to improve the intrauterine compromised condition of the fetal circulation.

\section{Case Reports}

\section{Case 1}

A 39-year-old Japanese primigravida woman was referred to our maternity center hospital at $24+2$ weeks of gestation due to oligohydramnios. She presented with a complicated uterine myoma and ulcerative colitis. There were no signs of any rupture of the membranes, such as discharge or bleeding.

Due to the small amount of amniotic fluid in the uterine cavity (amniotic fluid pocket (AP) $7 \mathrm{~mm}$ ) [5] when she presented to our hospital, it was not possible to perform a thorough ultrasonography examination to assess the fetus, umbilical cord and placenta. Thus, amnioinfusion was performed in an attempt to improve the visualization of the ultrasound scan. This led to the detection of the cause of the oligohydramnios. The amniotic cavity was injected with $725 \mathrm{~mL}$ of normal saline transabdominally by an experienced obstetrician under ultrasound guidance. After the amnioinfusion, the AP increased to $26 \mathrm{~mm}$. The fetus, umbilical cord and placenta could then be easily evaluated by ultrasound.

The ultrasound revealed a normal growing fetus (estimated fetal weight (EFW) $625 \mathrm{~g}$ ) without any morphological abnormalities. However, a hyper-coiled cord was revealed based on the antenatal coiling index [5] which was 0.84 (1/length of one cycle (cm)) (Fig. 1). Following the ultrasound, fetal heart tracing did not indicate either umbilical blood flow obstruction or a non-reassuring fetal status. Furthermore, no symptoms of ROM were observed after the amnioinfusion. Interestingly, after the procedure, oligohydramnios was not observed until delivery. The woman delivered a normal infant (weight: 2,777 g) transvaginally at $37+1$ weeks' gestation. The placenta weighed $460 \mathrm{~g}$, the length of the umbilical cord was $65 \mathrm{~cm}$ and a hyper-coiled cord was observed.

\section{Case 2}

This case was 45-year-old Japanese primiparous woman who 


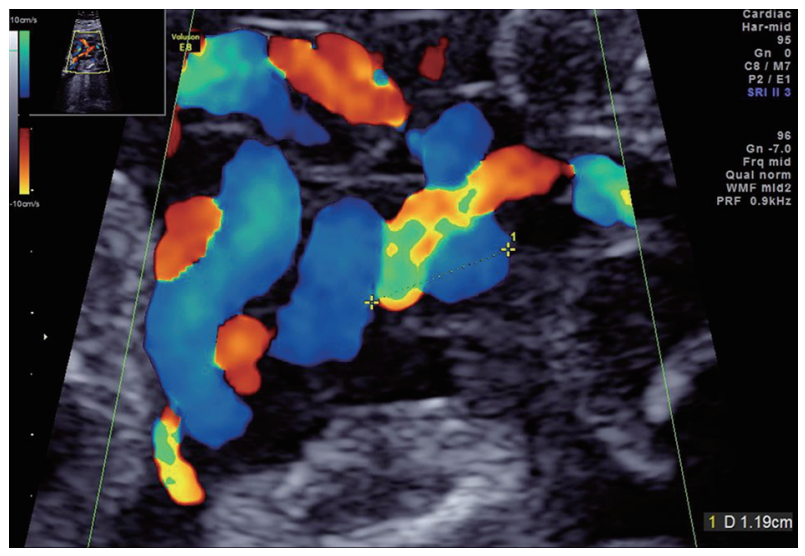

Figure 1. The ultrasonography findings of a hyper-coiled umbilical cord in case 1.

had a history of myomectomy for three myomas and adhesive intestinal obstruction. She achieved pregnancy by in vitro fertilization and embryo transfer. On ultrasound assessment at 20 +1 weeks' gestation in our hospital, the estimated fetal weight was $210 \mathrm{~g}$, which was - 2.0 standard deviation (SD) based on the Japanese standard [6], a hyper-coiled cord was detected by the coiling index of 0.6 , and marginal cord insertion of the placenta was revealed (Fig. 2).

She visited our outpatient clinic at $22+2$ weeks' gestation due to decreased fetal movement and fetal growth restriction. The volume of the amniotic fluid expressed by the amniotic pocket was decreased to $10.5 \mathrm{~mm}$. Ultrasound Doppler assessment revealed the reversed wave of ductus venosus and the pulsation of umbilical venous blood flow (Fig. 3a, b). She was therefore admitted to our hospital to monitor the well-being of the fetus.

It was hypothesized that the fetal growth restriction and the oligohydramnios may have resulted from umbilical cord abnormalities, such as a hyper-coiled cord and marginal cord insertion. After admission, her amniotic fluid volume was gradually increased to $22 \mathrm{~mm}$ after 2 weeks by only bed rest. The Doppler assessment of both the ductus venosus and umbilical venous flow also improved (Fig. 4a, b).

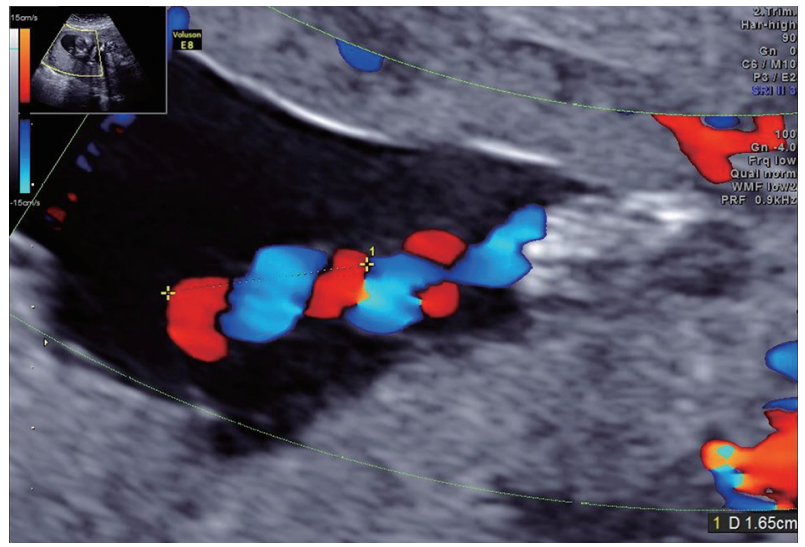

Figure 2. The ultrasonography findings of hyper-coiled umbilical cord in case 2.

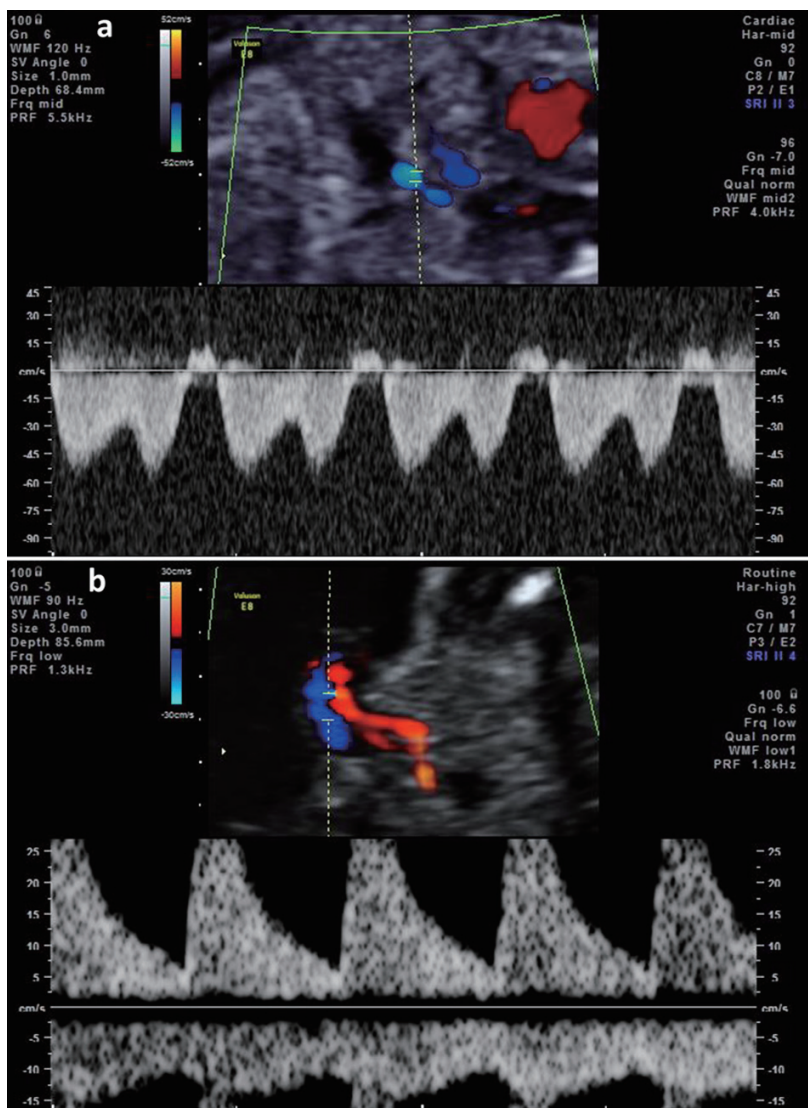

Figure 3. (a) The reversed wave of ductus venosus before the admission of case 2. (b) The umbilical venous pulsation before the admission of case 2 .

The well-being of the fetus was monitored by the assessments of both the Doppler blood flow and fetal heart tracing during pregnancy. At $37+3$ weeks' gestation, she delivered a neonate weighing $1,923 \mathrm{~g}(-2.3 \mathrm{SD})$ by cesarean section due to her history of a previous uterine operation. The placenta weighed $410 \mathrm{~g}$ with lobed placenta, marginal cord insertion and $70 \mathrm{~cm}$ of hyper-coiled cord. Calcification, infarction and fibrin deposition were observed in a pathological examination of the placenta. All procedures followed were in accordance with the ethical standards of the responsible committee on human experimentation and with the Helsinki Declaration of 1975, as revised in 2008 (5).

\section{Discussion}

An umbilical vessel of hyper-coiled cord tends to easily be inflected and obstructed by fetal movement or external force $[7,8]$. It may occur due to acute and chronic circulatory insufficiency. Acute blood obstruction of the umbilical vessels leads to decreased blood pressure and hypoxia resulting in a non-reassuring fetal status or intrauterine fetal death, whereas the growth and development of the fetus would be adversely affected in cases of temporary and mild obstruction.

It is hypothesized that the oligohydramnios status of case 

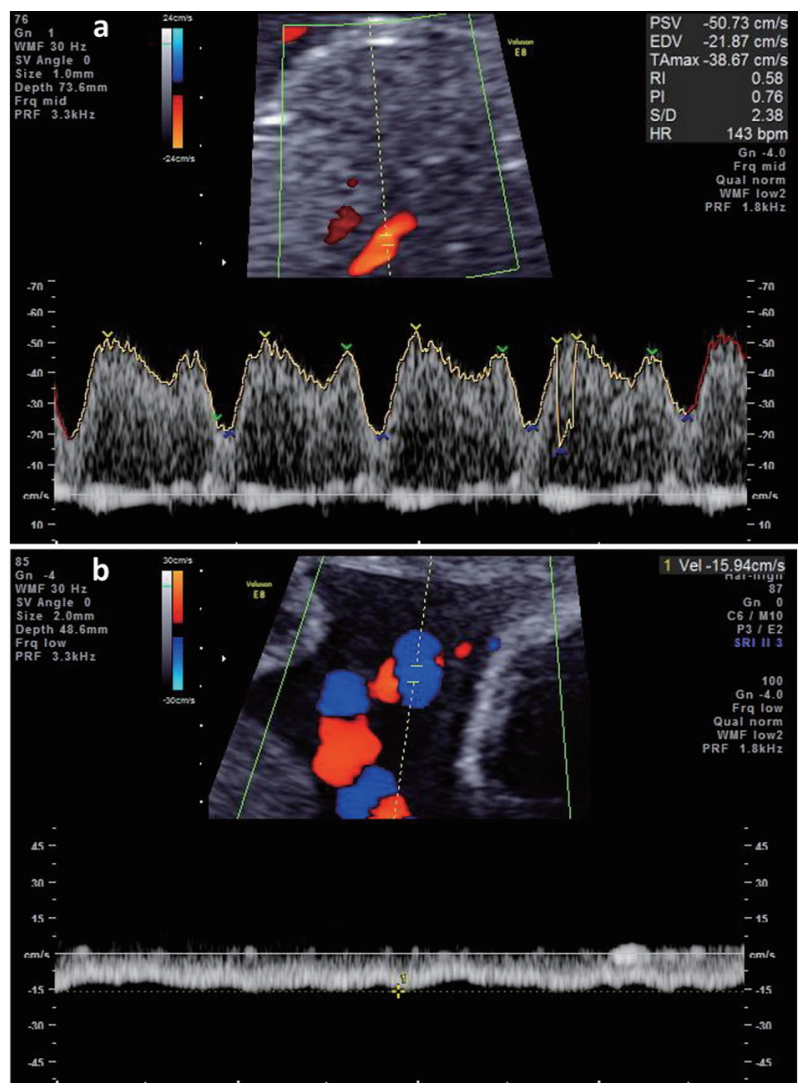

Figure 4. (a) The reversed wave of ductus venosus after the admission of case 2. (b) The umbilical venous pulsation after the admission of case 2.

1 occurred due to acute circulatory insufficiency caused by the hyper-coiled cord. However, the normal fetal growth indicated the absence of chronic circulatory insufficiency. As a consequence, amnioinfusion successfully improved the umbilical circulation by loosening the compression of the umbilical vessels, although our initial intention was simply to assess the fetus and the intrauterine structures by increasing the space in the uterine cave.

On the other hand, an interesting phenomenon was observed in case 2 . The patient's oligohydramnios improved with only bed rest, and without amnioinfusion. This phenomenon might indicate that umbilical circulatory insufficiency was remedied by the reduction of some of the uterine contractions. Fetal and umbilical circulation might gradually improve during pregnancy after admission.

A stillbirth caused by the sudden obstruction of the umbilical cord circulation due to hyper-coiled cord is not a rare event, even in fetuses that exhibit normal growth $[1,2,9]$. If the ultrasound findings led to the suspicion that the fetus with a hyper-coiled cord was exposed to severe conditions, such as end-diastolic absent or reversed flow in umbilical artery, ductus venosus or pulsation in umbilical vein [10], then emergency cesarean delivery could be selected to avoid sudden accidental intrauterine fetal death. On the other hand, in cases where the gestation is still in the early immature period, amniocentesis or bed rest may be a more useful option than cesarean section. However, it would be difficult to rescue an extremely immature fetus in cases where growth is severely restricted with chronic irreversible circulatory insufficiency due to hyper-coiled cord.

In the two present cases, the fetal pathophysiological evaluation based on both the AP and Doppler findings was found to improve by amnioinfusion and bed rest in the second trimester. However, there are some limitations associated with the explanation of this phenomenon because these improvements in the fetal circulation associated with a hyper-coiled cord were only observed by Doppler ultrasonography and we therefore speculated the patient's condition based on our experience. Accumulated experience was needed to reveal the evidence of these phenomena in the present report.

In conclusion, we experienced two cases in which the fetal-umbilical-placental circulation improved after amnioinfusion and/or bed rest. Amnioinfusion can be effective for releasing the compression of the umbilical vessels by forming a space around the umbilical cord in cases of oligohydramnios with an abnormal umbilical cord. Bed rest may also have a positive effect in reducing the compression of the umbilical vessels in such cases.

\section{Acknowledgments}

We would like to thank all doctors who managed these two cases for submitting this case report to JMC.

\section{Financial Disclosure}

There is no financial disclosure or funding of this case report.

\section{Conflict of Interest}

The authors declare that there is no conflict of interest.

\section{Informed Consent}

Informed consents were obtained from all patients for being included in the study.

\section{Author Contributions}

$\mathrm{MN}$ and $\mathrm{JH}$ conceived the study, MN analyzed the data, and $\mathrm{MN}, \mathrm{JH}$ and $\mathrm{HT}$ managed these two cases, $\mathrm{MN}$, JH and $\mathrm{AS}$ wrote the first draft of the manuscript. All authors collected data.

\section{Data Availability}

The data supporting the findings of this study are available from the corresponding author upon reasonable request. 


\section{References}

1. Ernst LM, Minturn L, Huang MH, Curry E, Su EJ. Gross patterns of umbilical cord coiling: correlations with placental histology and stillbirth. Placenta. 2013;34(7):583588.

2. Hasegawa J, Matsuoka R, Ichizuka K, Sekizawa A, Okai T. Ultrasound diagnosis and management of umbilical cord abnormalities. Taiwan J Obstet Gynecol. 2009;48(1):23-27.

3. Skulstad SM, Rasmussen S, Iversen OE, Kiserud T. The development of high venous velocity at the fetal umbilical ring during gestational weeks 11-19. BJOG. 2001;108(3):248-253.

4. Ghosh A, Woo JS, MacHenry C, Wan CW, O'Hoy KM, Ma HK. Fetal loss from umbilical cord abnormalities-a difficult case for prevention. Eur J Obstet Gynecol Reprod Biol. 1984;18(4):183-198.

5. Bastide A, Manning F, Harman C, Lange I, Morrison
I. Ultrasound evaluation of amniotic fluid: outcome of pregnancies with severe oligohydramnios. Am J Obstet Gynecol. 1986;154(4):895-900.

6. Itabashi K, Miura F, Uehara R, Nakamura Y. New Japanese neonatal anthropometric charts for gestational age at birth. Pediatr Int. 2014;56(5):702-708.

7. Benirschke K. Obstetrically important lesions of the umbilical cord. J Reprod Med. 1994;39(4):262-272.

8. Dodds M, Windrim R, Kingdom J. Complex umbilical cord entanglement. J Matern Fetal Neonatal Med. 2012;25(9):1827-1829.

9. Tantbirojn P, Saleemuddin A, Sirois K, Crum CP, Boyd TK, Tworoger S, Parast MM. Gross abnormalities of the umbilical cord: related placental histology and clinical significance. Placenta. 2009;30(12):1083-1088.

10. Clerici G, Antonelli C, Rizzo G, Kanninen TT, Di Renzo GC. Atypical hemodynamic pattern in fetuses with hypercoiled umbilical cord and growth restriction. J Matern Fetal Neonatal Med. 2013;26(6):558-562. 